\title{
On Embedding Trees in Grids
}

\author{
Vitor Tocci Ferreira de Luca ${ }^{1}$, Fabiano de Souza Oliveira ${ }^{1}$, Jayme Luiz Szwarcfiter ${ }^{1,2}$ \\ ${ }^{1}$ Instituto de Matemática e Estatística - Universidade do Estado do Rio de Janeiro (UERJ) \\ CEP 20550-900 - Rio de Janeiro - RJ - Brazil \\ ${ }^{2}$ COPPE, NCE, IM - Universidade Federal do Rio de Janeiro (UFRJ) \\ CEP 21941-600 - Rio de Janeiro - RJ - Brazil \\ toccivitor8@gmail.com, fabiano.oliveira@ime.uerj.br, jayme@nce.ufrj.br
}

\begin{abstract}
We are interested in embedding trees $T$ with $\Delta(T) \leq 4$ in a rectangular grid, such that the vertices of $T$ correspond to grid points, while edges of $T$ correspond to non-intersecting straight segments of the grid lines. The aim is to minimize the maximum number of bends of a path of $T$. We provide a quadratictime algorithm for this problem. By applying this algorithm, we obtain an upper bound on the number of bends of EPG representations of VPT $\cap$ EPT graphs.
\end{abstract}

\section{Introduction}

The problem of grid embedding is that of drawing a graph $G$ onto a rectangular twodimensional grid (called simply grid) such that each vertex $v \in V(G)$ corresponds to a grid point (an intersection of a horizontal and a vertical grid line) and the edges of $G$ correspond to paths of the grid. Grid embedding of graphs has been considered with different perspectives [Beck and Storandt 2020, Liu et al. 1998, Schnyder 1990]. In [Liu et al. 1998], linear-time algorithms are described for embedding planar graphs having their edges drawn as non-intersecting paths in the grid, such that the maximum number of bends of any edge is minimized, as well as the total number of bends.

In this paper, we are interested in a variation of this problem: given a planar graph $G$, find a grid embedding of $G$ such that the edges of $G$ correspond to pairwise non-intersecting paths of the grid, having no bends. The aim is to minimize the maximum number of bends of any path in the embedding, over all paths of $G$. For instance, the tree in Figure 1(a) is drawn in such a way that there is a path having 5 bends (the path joining $o$ and $m$ ), and 5 is the maximum number of bends in that drawing. However, this maximum number of bends can be decreased to 3 bends (path joining $e$ and $f$ ), as Figure 1(b) illustrates.

A motivation for this problem lies in finding certain grid representations of VPT $\cap$ EPT graphs, as defined next. Given a tree $T$, called host tree, and a set $\mathcal{P}$ of nontrivial paths in $T$, the vertex (resp. edge) intersection graph of paths in a tree (VPT (resp. EPT)) of $\mathcal{P}$ is the graph denoted by $\operatorname{VPT}(\mathcal{P})(\operatorname{resp}$. $\operatorname{EPT}(\mathcal{P}))$ having $\mathcal{P}$ as vertex set and two vertices adjacent if the corresponding paths have in common at least one vertex (resp. edge). We say that $\langle T, \mathcal{P}\rangle$ is a VPT (resp. EPT) representation of $G$.

Instead of trees, the graphs we are interested have a grid $\mathcal{G}$ as the underlying structure from which a collection of nontrivial paths $\mathcal{P}$ is considered. The edge intersection

The first author is supported by CAPES. Second author is partially supported by FAPERJ. Third author is partially supported by FAPERJ and CNPq. 


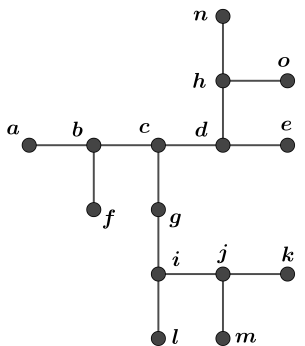

(a)

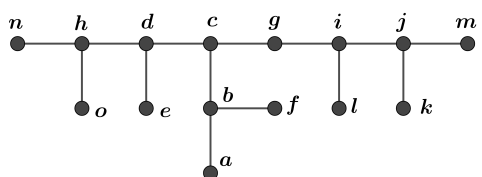

(b)

Figure 1. Two possible models $\mathcal{M}_{1}$ (left) and $\mathcal{M}_{2}$ (right) of the same tree $T$.

graph of paths of a grid of $\mathcal{P}$ is the graph denoted by $\operatorname{EPG}(\mathcal{P})$ having $\mathcal{P}$ as vertex set and two vertices adjacent if the corresponding paths have in common at least one edge of the grid. We say that $\mathcal{P}$ is an EPG representation of $G$. A turn of a path at a grid point is called a bend and the grid point in which a bend occurs is called a bend point. An EPG representation is a $B_{k}-E P G$ representation if each path has at most $k$ bends. A graph that admits a $\mathrm{B}_{k}$-EPG representation is called $\mathrm{B}_{k}$-EPG.

\section{Embedding trees in a grid}

Let $T$ be a tree such that $\Delta(T) \leq 4$. Consider the problem of embedding such a tree in a grid $\mathcal{G}$, so that the vertices must be placed at grid points and the edges drawn as non-intersecting paths of $\mathcal{G}$ with no bends, which we will call linear embedding of $T$, or simply a model of $T$. Figure 1 depicts two possible models corresponding to a same tree. Given a path $Q=v_{1}, v_{2}, \ldots, v_{k}$ of $T$, and a model $\mathcal{M}$ of $T$, the number of bends of $Q$ is defined to be the number of bends of the grid path consisting of the concatenation of paths of the grid corresponding to the edges $\left(v_{1}, v_{2}\right),\left(v_{2}, v_{3}\right), \ldots,\left(v_{k-1}, v_{k}\right)$ in $\mathcal{M}$. In Figure 1(a), the path $o, h, d, c, g, i, j, m$ has 5 bends, and only 1 bend in Figure 1(b).

Among all possible models, consider the problem of finding one in which the maximum number of bends of a path of $T$, over all of them, is minimum. Note that, since every path of a tree is contained in a leaf-to-leaf path, a path that bends the most in a given model is a leaf-to-leaf path, and therefore those are the only ones to be considered. More formally, let $\mathcal{M}(T)$ be the set of all possible models of a given tree $T$ and $u, v \in V(T)$ be leaves of $T$. The number of bends of the path connecting $u$ and $v$ in a model $\mathcal{M} \in \mathcal{M}(T)$ is denoted by $b_{\mathcal{M}}(u, v)$. Therefore, $b_{\mathcal{M}_{1}}(o, m)=5$ and $b_{\mathcal{M}_{2}}(o, m)=1$. Let

$$
b(\mathcal{M})=\max \left\{b_{\mathcal{M}}(u, v) \mid u \text { and } v \text { are leaves in } T\right\}
$$

denote the number of bends of the leaf-to-leaf path that bends the most in $\mathcal{M}$, and

$$
b(T)=\min \{b(\mathcal{M}) \mid \mathcal{M} \in \mathcal{M}(T)\}
$$

the minimum number of bends of a model, over all of them. Figure 1(a) depicts a model $\mathcal{M}_{1}$ of a tree $T$ such that $b\left(\mathcal{M}_{1}\right)=5$, therefore $b(T) \leq 5$. Figure 1(b) shows another model $\mathcal{M}_{2}$ for which $b\left(\mathcal{M}_{2}\right)=3$ and, therefore, $b(T) \leq 3$. It is possible to show that no model $\mathcal{M}$ of $T$ has $b(\mathcal{M})=2$ and, therefore, $b(T)=3$. Given $\mathcal{M} \in \mathcal{M}(T)$, let $b_{\mathcal{M}}^{\ell}(p, v)$ denote the maximum number of bends found in a single path having as extreme vertices $p$ and a leaf of $T$, over all paths that contain $v \in V(T)$. That is,

$$
b_{\mathcal{M}}^{\ell}(p, v)=\max \left\{b_{\mathcal{M}}(p, f) \mid f \text { is a leaf of } T \text { and the path } p, \ldots, f \text { contains } v\right\} .
$$


Also, define

$$
b_{T}^{\ell}(p, v)=\min \left\{b_{\mathcal{M}}^{\ell}(p, v) \mid \mathcal{M} \in \mathcal{M}(T)\right\} .
$$

Let $\mathcal{M} \in \mathcal{M}(T)$ and $v \in V(T)$. Let $\left\{u_{\mathcal{M}}^{i}(v) \mid 1 \leq i \leq d(v)\right\}$ be $N(v)$ and $b_{\mathcal{M}}^{i}(v)=b_{\mathcal{M}}^{\ell}\left(v, u_{\mathcal{M}}^{i}(v)\right)$. For $d(v)<i \leq 4$, define "virtual" neighbors $u_{\mathcal{M}}^{i}(v)=\emptyset$ for which $b_{\mathcal{M}}^{i}(v)=-1$. Assume that the neighbors (both real and virtual) are ordered so that $b_{\mathcal{M}}^{i}(v) \geq b_{\mathcal{M}}^{i+1}(v)$ for all $1 \leq i<4$. As examples, $u_{\mathcal{M}_{2}}^{1}(i)=g$ (and $b_{\mathcal{M}_{2}}^{1}(i)=2$ ), $u_{\mathcal{M}_{2}}^{2}(i)=j$ (and $b_{\mathcal{M}_{2}}^{2}(i)=1$ ), $u_{\mathcal{M}_{2}}^{3}(i)=l$ (and $b_{\mathcal{M}_{2}}^{3}(i)=0$ ), and $u_{\mathcal{M}_{2}}^{4}(i)=\emptyset$ (and $\left.b_{\mathcal{M}_{2}}^{4}(i)=-1\right)$. We say that $v$ is balanced if $u_{\mathcal{M}}^{1}(v)$ and $u_{\mathcal{M}}^{2}(v)$ are mutually in the same horizontal or vertical grid line in $\mathcal{M}$ (and, therefore, so are $u_{\mathcal{M}}^{3}(v)$ and $u_{\mathcal{M}}^{4}(v)$ ).

The algorithm consists of the following steps. First, let $v_{0}, v_{1}, \ldots, v_{n-1}$ be a sequence of $V(T)$ such that each $v_{i}$ is adjacent to exactly one vertex $p_{i}$ for all $1 \leq i<n$, where $p_{i}=v_{j}$ for some $0 \leq j<i$. Let $\mathcal{M}$ be a model having a single vertex $v_{0}$ at some grid point. For $i=1,2, \ldots, n-1$, add to $\mathcal{M}$ the vertex $v_{i}$ attached to the grid point of $p_{i}$, in any free horizontal or vertical grid line of $p_{i}$. Then, call the procedure of balancing $v_{i}$. Such a procedure consists of traversing $T$ rooted at $v_{i}$ in post-order. The operation of visiting a vertex $v$ consists of making $v$ balanced by rearranging in $\mathcal{M}$ the drawing of the four subtrees of $v$ rooted at $u_{\mathcal{M}}^{i}(v)$ (for $1 \leq i \leq 4$ ), potentially rotating and rescaling them to fit. Regarding the time complexity of the algorithm, it is possible to keep the values of $u_{\mathcal{M}}^{i}(v)$ stored for each $v \in V(T)$ and $1 \leq i \leq 4$, and update them right after the balance step in constant time, based on which subtrees had their positions exchanged, and on the respective values of $u_{\mathcal{M}}^{i}(w)$ from the neighbors $w \in N(v)$. Since the algorithm performs $n-1$ post-order traversals in $T$, the algorithm runs in $O\left(n^{2}\right)$ time.

Theorem 1. Given a tree $T$, let $\mathcal{M}$ be the model produced by the execution of the algorithm on input $T$. Then, $b(\mathcal{M})=b(T)$.

\section{EPG representations of VPT $\cap$ EPT graphs}

We provide an upper bound on the number of bends of an EPG representation of VPT $\cap$ EPT graphs. The VPT $\cap$ EPT graphs are those that can be represented in host trees with maximum degree at most 3 [Golumbic and Jamison 1985]. In [Alcón et al. 2015], this class is characterized by a family of minimal forbidden induced subgraphs.

Let $G$ be a VPT graph and $\langle T, \mathcal{P}\rangle$ a VPT representation of $G$. Consider $V(G)=$ $\left\{v_{1}, v_{2}, \ldots, v_{n}\right\}, V(T)=\left\{u_{1}, u_{2}, \ldots, u_{m}\right\}$ and $\mathcal{P}=\left\{Q_{i} \mid v_{i} \in V(G)\right\}$. Build an EPG representation $\mathcal{R}=\left\{P_{i} \mid v_{i} \in V(G)\right\}$ of $G$ in a grid $\mathcal{G}$ in the following way.

First, let $\mathcal{M}$ be a model of $T$ with the minimum number of bends on the grid $\mathcal{G}$, as described in Section 2. For all edges $e_{i}$ of $T$ in $\mathcal{M}$, let $e_{i}^{\prime}$ be their midpoints in grid $\mathcal{G}$. For all $u_{i} \in V(T)$ such that $d\left(u_{i}\right)=1$, build an auxiliary path, $P_{u_{i}}^{\prime}$, going from $u_{i}$ to $e^{\prime}$, where $e$ is the edge to which $u_{i}$ is incident. For all $u_{i} \in V(T)$ such that $d\left(u_{i}\right)=2$, let $e_{1}$ and $e_{2}$ be the edges incident to $u_{i}$. Build an auxiliary path $P_{u_{i}}^{\prime}$ having $e_{1}^{\prime}$ and $e_{2}^{\prime}$ as endpoints. For all $u_{i} \in V(T)$ such that $d\left(u_{i}\right)=3$, let $e_{1}, e_{2}$ and $e_{3}$ be the edges incident to $u_{i}$. Note that, at least one of them is vertical and at least one of them is horizontal. Without loss of generality, assume $e_{1}$ is vertical and $e_{2}$ is horizontal. Build an auxiliary path $P_{u_{i}}^{\prime}$ having $e_{1}^{\prime}$ and $e_{2}^{\prime}$ as endpoints. For all $Q_{i} \in \mathcal{P}$, let $u_{i}$ be an endpoint of $Q_{i}$. Initialize $P_{i}$ to be coincident to $Q_{i}$. Next, consider the following cases:

- if $d\left(u_{i}\right)=2$, enlarge $P_{i}$ by stretching its endpoint so that it coincides with the endpoint of $P_{u_{i}}^{\prime}$ that does not belong to $P_{i}$ yet. 
- If $d\left(u_{i}\right)=3$ and $P_{i} \cap P_{u_{i}}^{\prime}=\left\{u_{i}\right\}$, enlarge $P_{i}$ by stretching its endpoint so that it coincides with the endpoint of $P_{u_{i}}^{\prime}$ which does not impose a new bend in $P_{i}$.

- If $d\left(u_{i}\right)=3$ and $P_{i} \cap P_{u_{i}}^{\prime} \neq\left\{u_{i}\right\}$, it implies that $u_{i}$ is an endpoint of $P_{i}$ and $P_{i}$ already contains one of the endpoints of $P_{u_{i}}^{\prime}$. In that case, enlarge $P_{i}$ by stretching its endpoint so that it coincides with the other endpoint of $P_{u_{i}}^{\prime}$.

Remove the paths $P_{u_{i}}^{\prime}$ for all $1 \leq i \leq m$. Such a construction builds a $\mathrm{B}_{k}$-EPG representation of $G$ with $k \leq b(T)$. Note that, if a path $Q_{i}$ with $u_{i}$ as an extreme vertex has $b(T)$ bends, then $d\left(u_{i}\right) \leq 2$. Therefore, either $P_{i}=Q_{i}$ or $P_{i}$ is $Q_{i}$ with their extreme vertices stretched without any new bends. Thus, $P_{i}$ has $b(T)$ bends and, therefore, $\mathcal{R}$ has a maximum of $b(T)$ bends in any of its paths. Figure 2(b) presents an EPG representation $\mathcal{R}=\left\{P_{i} \mid 1 \leq i \leq 10\right\}$ derived for the family $\mathcal{P}=\left\{Q_{i} \mid 1 \leq i \leq 10\right\}$ of Figure 2(a).

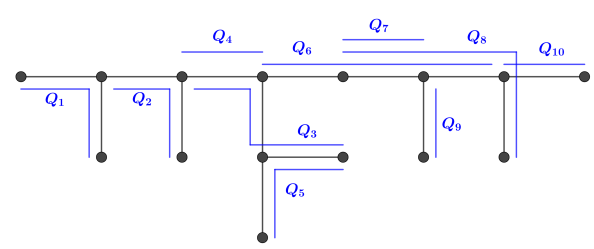

(a)

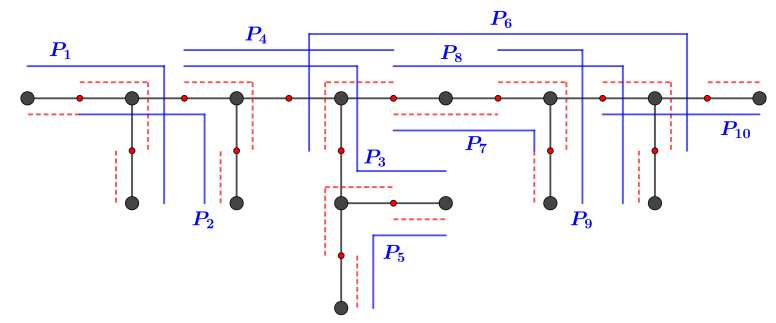

(b)

Figure 2. Construction of a $\mathbf{B}_{k}$-EPG representation with $k \leq b(T)$.

\section{Conclusion}

In this paper, we presented an algorithm to embed a tree $T$ with $\Delta(T) \leq 4$ in a rectangular grid, such that the maximum number of bends of any path of $T$ is minimized. We also described how to use such models to construct EPG representations of VPT $\cap$ EPT graphs providing an upper bound on the number of bends of such graphs.

\section{References}

Alcón, L., Gutierrez, M., and Mazzoleni, M. P. (2015). Characterizing paths graphs on bounded degree trees by minimal forbidden induced subgraphs. Discrete Mathematics, 338(1):103-110.

Beck, M. and Storandt, S. (2020). Puzzling grid embeddings. In 2020 Proceedings of the Twenty-Second Workshop on Algorithm Engineering and Experiments (ALENEX), pages 94-105. SIAM.

Golumbic, M. C. and Jamison, R. E. (1985). Edge and vertex intersection of paths in a tree. Discrete Mathematics, 55(2):151-159.

Liu, Y., Morgana, A., and Simeone, B. (1998). A linear algorithm for 2-bend embeddings of planar graphs in the two-dimensional grid. Discrete Applied Mathematics, 81(13):69-91.

Schnyder, W. (1990). Embedding planar graphs on the grid. In Proceedings of the first annual ACM-SIAM symposium on Discrete algorithms, pages 138-148. 